PESQUIMAT, Revista de la Fac.CC.MM. de la

UNIVERSIDAD NACIONAL MAYOR DE SAN MARCOS

Vol.IV, $N^{\circ} 2$, pág.67-75, LIMA-PERÚ. Diciembre 2001

\title{
OBSERVACIONES SOBRE LA EXISTENCIA GLOBAL DE LAS SOLUCIONES DE UNA ECUACION DE KIRCHOFF NO LINEAL VIA EL METODO DE TARTAR
}

\author{
E. Cabanillas, J. Bernui \& Z. Huaringa ${ }^{1}$
}

\begin{abstract}
En el presente trabajo investigamos la existencia global de las soluciones de una Ecuación no Lineal, prescindiendo del "Potential well Method" y aplicamos el método del Tartar
\end{abstract}

\section{INTRODUCCIÓN *}

En este trabajo estudiamos la existencia global y el decaimiento de la enegía del Sistema no Lineal:

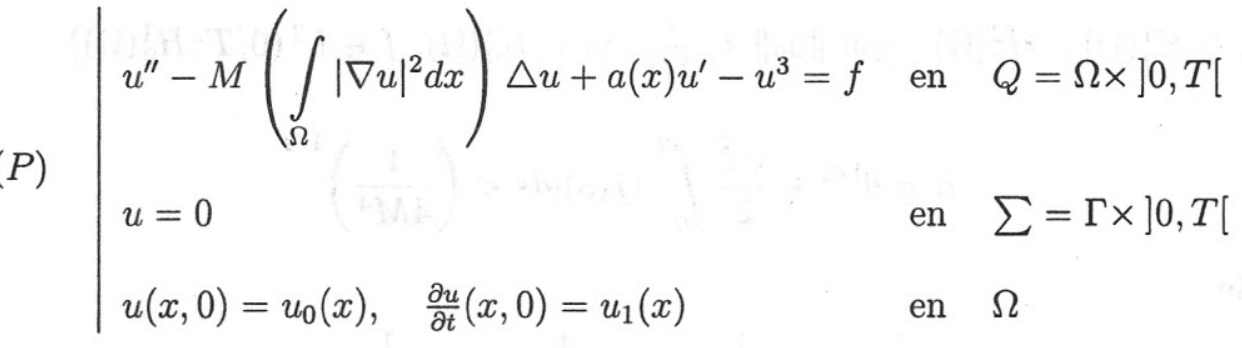

donde $\Omega$ es un abierto acotado de $\mathbb{R}^{3}$, bien regular, de frontera $\Gamma, T>0, M(s)=1+s$, $\forall s \geq 0, \Delta$ es el operador de Laplace; $a$ y $f$ son funciones dadas.

La existencia global para el Sistema $(P)$ en el caso $a(x)=c$ (constante positiva) ha sido investigado por diversos autores: Ono [3], Matsuyama-Ikehata [2], FeitosaDueñas [1], etc. Todos ellos usaron el método del "Potencial Well" en su análisis; nosotros usaremos el método de Tartar [4]. Nuestro estudio fue motivado al investigar la estabilización del Sistema $(P)$ cuando el coficiente disipativo está localizado en una vecindad de la frontera por lo que creemos nuestro resultado es de importancia.

\footnotetext{
${ }^{1}$ Universidad Nacional Mayor de San Marcos. Facultad de Ciencias Matemáticas.
} 


\section{PRELIMINARES}

Con $(.,),. \mid$. | denotaremos el potencial el producto interno y la norma de $L^{2}(\Omega)$ respectivamente, $H^{m}(\Omega)$ es el espacio de Sobolev usual. Dotamos al espacio $H_{0}^{1}(\Omega)$ con la norma del gradiente

$$
\|u\|=|\nabla u|, \forall u \in H_{0}^{1}(\Omega)
$$

Lemma 1.1. (Sobolev). Sea $1 \leq q \leq 6$. Entonces se verifica

$$
|u|_{q} \leq C_{q}\|u\|, \forall u \in H_{0}^{1}(\Omega)
$$

donde | | representa la norma usual de $L^{2}(\Omega)$

\section{EL RESULTADO PRINCIPAL}

Teorema 3.1. Sea $a \in L^{\infty}(\Omega) \cap C^{1}(\Omega)$ tal que:

$$
a(x) \geq a_{0}>0, \forall x \in \Omega ;|\triangle a(x)| \in C_{0} a(x),|\nabla a(x)|^{2} \leq C_{1} a(x)
$$

donde $C_{0}$ y $C$, son constantes positivas.

Si $u_{0} \in H_{0}^{1}(\Omega) \cap H^{2}(\Omega)$, con $\left\|u_{0}\right\|<\frac{1}{M^{2}}, u_{1} \in H_{0}^{1}(\Omega), f \in L^{2}\left(0, T ; H_{0}^{1}(\Omega)\right)$

$$
\alpha=\theta^{1 / 2}+\frac{\sqrt{2}}{2} \int_{0}^{T}|f(s)| d s<\left(\frac{1}{4 M^{4}}\right)^{1 / 2}
$$

donde

$$
\begin{gathered}
\theta=\frac{1}{2}\left|u_{1}\right|^{2}+\frac{1}{2}\left\|u_{0}\right\|^{2}+\frac{1}{4}\left\|u_{0}\right\|^{4}-\frac{1}{4}\left|u_{0}\right|_{4}^{4} y \\
p(\lambda)=\frac{1}{2} \lambda^{2}-\frac{\left|c_{4}-1\right|}{4} \lambda^{4}
\end{gathered}
$$

entonces existe un número positivo $\epsilon_{0}$ tal que si:

$$
\left\|u_{1}\right\|+\left|\triangle u_{0}\right|<\epsilon_{0}
$$

entonces existe una función $u: \Omega \times] 0, T[\longrightarrow \mathbb{R}$, tal que 


$$
u \in L^{\infty}\left(0, T ; H_{0}^{1}(\Omega) \cap H^{2}(\Omega)\right)
$$

(3.3) $\quad u^{\prime} \in L^{\infty}\left(0, T ; H_{0}^{1}(\Omega)\right)$

(3.4) $\quad u^{\prime \prime} \in L^{\infty}\left(0, T ; L^{2}(\Omega)\right)$

$$
\frac{d}{d t}\left(u^{\prime}(t), w\right)+\left(1+|\nabla u(t)|^{2}\right)(-\triangle u(t), w)+\left(a(x) u^{\prime}(t), w\right)
$$$$
-\left(u^{3}(t), w\right)=(f(t), w) \forall w \in H_{0}^{1}(\Omega) \text {, en el sentido de } D^{\prime}(0, T)
$$

(3.6) $u(0)=u_{0}, u^{\prime}(0)=u_{1}$

Demostración. Usaremos el método de Faedo-Galerkin.

Sea $\left\{w_{1}, w_{2}, \cdots, w_{j}, \cdots\right\}$ una base Hilbertiana de $H_{0}^{1}(\Omega) \cap H^{2}(\Omega)$ formada por los vectores propios de $-\triangle$ esto es:

$$
\mid \begin{array}{cc}
-\triangle w_{j}=\lambda_{j} w_{j} & \text { en } \Omega \\
w_{j}=0 & \text { en } \Gamma
\end{array}
$$

Consideremos $V_{m}=\left[w_{1}, w_{2}, \cdots, w_{m}\right]$ el subespacio de $H_{0}^{1}(\Omega)$ generado por $\left\{w_{1}\right.$, $\left.w_{2}, \cdots, w_{m}\right\}$. Buscamos una solución $u_{m}(t)$ de la forma:

$$
u_{m}(t)=\sum_{j=1}^{m} g_{j_{m}}(t) w_{j}
$$

donde $g_{j_{m}}$ están determinadas por el sistema:

$$
\begin{array}{ll}
(3.7) \quad\left(u_{m}^{\prime \prime}(t), w\right)-\left(1+\left|\nabla u_{m}(t)\right|^{2}\right)\left(\triangle u_{m}(t), w\right)+ \\
\\
\left(a(x) u_{m}^{\prime}(t), w\right)-\left(u_{m}^{3}(t), w\right)=(f(t), w) ; \forall w \in V_{m} \\
\text { (3.8) } \quad u_{m}(0)=u_{0 m} \longrightarrow u_{0} \text { en } H_{0}^{1}(\Omega) \cap H^{2}(\Omega) \\
\text { (3.9) } \quad u_{m}^{\prime}(0)=u_{1 m} \longrightarrow u_{1} \text { en } H_{0}^{1}(\Omega)
\end{array}
$$


Por el teorema de Caratheodory existe una solución local $u_{m}(t)$ en $\left[0, T_{m}[\right.$. Las estimativas a priori permiten extender la solución al intervalo $[0, T]$ independiente de $m$

Estimativa a Priori I

Para $w=u_{m}^{\prime}(t)$ en (3.7) se tiene:

$$
\begin{gathered}
\frac{d}{d t}\left\{\frac{1}{2}\left|u_{m}^{\prime}(t)\right|^{2}+\frac{1}{2}\left\|u_{m}(t)\right\|^{2}+\frac{1}{4}\left\|u_{m}(t)\right\|^{4}-\frac{1}{4} \mid u_{m}(t) \|_{4}^{4}\right\} \\
+\int_{\Omega} a(x)\left[u_{m}{ }^{\prime}(t)\right]^{2} d x=\left(f(t), u_{m}{ }^{\prime}(t)\right)
\end{gathered}
$$

Ahora

Si $C_{4} \leq 1$ entonces $\left|u_{m}(t)\right|_{4} \leq\left\|u_{m}(t)\right\|$ y asi:

$$
0 \leq\left\|u_{m}(t)\right\|^{4}-\left|u_{m}(t)\right|_{4}^{4}
$$

Aplicando entonces el lema de Groniwall obtenemos:

$$
\left|u_{m}{ }^{\prime}(t)\right| \leq M_{1},\left\|u_{m}(t)\right\| \leq M_{1}
$$

Primero veamos un resultado previo.

Si $C_{4}>1$ entonces $|u(t)|^{4} \leq C_{4}^{4}\|u(t)\|^{4}$ y se tiene:

$$
\text { (3.11) } \frac{1}{2}\|u(t)\|^{2}-\frac{1}{4}\left(C_{4}-1\right)\|u(t)\|^{4} \leq \frac{1}{2}\|u(t)\|^{2}+\frac{1}{4}\|u(t)\|^{4}-\frac{1}{4}|u(t)|_{4}^{4}
$$

Sea $M^{4}=C_{4}-1>0$. Entonces el polinomio

$$
p(a)=\frac{1}{2} \lambda^{2}-\frac{M^{4}}{4} \lambda^{4}
$$

tiene raíces en $\lambda=0, \lambda= \pm \frac{\sqrt{2}}{M^{2}}$, los puntos de máximo son $\left( \pm \frac{1}{M^{2}}, \frac{1}{4 M^{4}}\right)$, $p(\lambda) \geq 0, \forall \lambda \in\left[-\frac{\sqrt{2}}{M^{2}}, \frac{\sqrt{2}}{M^{2}}\right]$ y $p(\lambda)$ es creciente en $\left.]-\infty,-\frac{1}{M^{2}}\right] \cup\left[0, \frac{1}{M^{2}}\right]$ 
De (3.11) resulta en particular para $\|u(t)\| \in\left[0, \frac{1}{M^{2}}\right]$ que:

$$
0 \leq p(\|u(t)\|) \leq \frac{1}{2}\|u(t)\|^{2}+\frac{1}{4}\|u(t)\|^{4}-\frac{1}{4}|u(t)|_{4}^{4}
$$

Vayamos ahora a la obtención de la estimativa. Por hipótesis

$$
\alpha^{2}<\frac{1}{4 M^{4}}
$$

Así, de la continuidad de $p$, existe $0<\beta<\frac{1}{M^{2}}$ tal que

$$
\frac{1}{2} \beta^{2}-\frac{1}{4} \beta^{4}=p(\beta)=\alpha^{2} .
$$

También $p\left(\left\|u_{0}\right\|\right) \geq 0 \mathrm{y}$

$$
\left\|u_{m}(0)\right\|<\frac{1}{M^{2}}, \text { para } m \text { suficientemente grande. }
$$

Probamos que:

$$
\left\|u_{m}(t)\right\|<\frac{1}{M^{2}}, \forall t \in\left[0, T_{m}[\right.
$$

Supongamos que existe $t_{0} \in\left[0, T_{m}[\right.$ tal que:

$$
\left\|u_{m}\left(t_{0}\right)\right\| \geq \frac{1}{M^{2}}
$$

Sea entonces:

$$
t^{*}=\inf \left\{t /\left\|u_{m}(t)\right\| \geq \frac{1}{M^{2}}\right\}
$$

De (3.13): $t^{*}>0$ y de la continuidad de las soluciones aproximadas obtenemos

$$
\begin{aligned}
& \left\|u_{m}\left(t^{*}\right)\right\|=\frac{1}{M^{2}} \\
& \left\|u_{m}(t)\right\| \leq \frac{1}{M^{2}}, \forall t \in\left[0, t^{*}\right]
\end{aligned}
$$

Hagamos 


$$
Z_{m}=\frac{1}{2}\left|u_{1 m}\right|^{2}+\frac{1}{2}\left\|u_{0 m}\right\|^{2}+\frac{1}{4}\left\|u_{0 m}\right\|^{4}-\frac{1}{4}\left|u_{0 m}\right|_{4}^{4}
$$

De (3.8) y (3.9)

$$
Z_{m} \longrightarrow \theta
$$

Ahora de (3.10), (3.12), (3.16), (3.17) y $p(\lambda) \geq 0$ en $\left[-\frac{1}{M^{2}}, \frac{1}{M^{2}}\right]$ :

$$
\begin{aligned}
\frac{1}{2}\left|u_{m}^{\prime}(t)\right|^{2} & \leq \frac{1}{2}\left|u_{m}{ }^{\prime}(t)\right|^{2}+p\left(\left\|u_{m}(t)\right\|\right)+\int_{0}^{t} \int_{\Omega} a(x)\left[u_{m}^{\prime}(s)\right]^{2} d x d s \\
& \leq Z_{m}+\int_{0}^{t}\left(f(s), u_{m}^{\prime}(s)\right) d s \\
& \leq \theta+\epsilon+\int_{0}^{t}|b(s)|\left|u_{m}^{\prime}(s)\right| d s \equiv \varphi(t), \forall \epsilon>0
\end{aligned}
$$

De la definición de la $\varphi$ se sigue que:

$$
\varphi^{1 / 2}(t) \leq \sqrt{\theta+\epsilon}+\frac{\sqrt{2}}{2} \int_{0}^{T}|f(s)| d s, \forall \epsilon>0
$$

lo que implica usando la hipótesis que:

$$
\varphi(t) \leq \alpha^{2}<\frac{1}{4 M^{4}} \quad \forall t \in\left[0, t^{\star}\right]
$$

De (3.18) y (3.19) se obtienen:

$$
p\left(\left\|u_{m}(t)\right\|\right) \leq \alpha^{2}, \quad \forall t \in\left[0, t^{\star}\right]
$$

De esto se sigue que

$$
\left\|u_{m}(t)\right\| \leq \dot{\beta}, \quad \forall t \in\left[0, t^{\star}\right]
$$

y así

$$
\left\|u_{m}\left(t^{\star}\right)\right\| \leq \beta<\frac{1}{M^{2}}
$$


lo que contradice (3.15), por lo que vale (3.14).

De (3.14) y (3.18) resulta:

$$
\left|u_{m}^{\prime}(t)\right| \leq \frac{\sqrt{2}}{2 M^{2}}, \quad \forall t \in\left[0, T_{m}[\right.
$$

En resumen hemos mostrado que:

$$
\left|u_{m}{ }^{\prime}(t)\right| \leq M_{0} \text { y }\left\|u_{m}(t)\right\| \leq M_{0}, \quad \forall t \in\left[0, T_{m}\right], M_{0}>0
$$

Estimativa a Priori II

Poniendo $w=-2 \Delta u_{m}^{\prime}(t)$ en (3.7) se tiene:

$$
\begin{aligned}
& \frac{d}{d t}\left\{\left|\nabla u_{m}^{\prime}(t)\right|^{2}+\left(1+\left|\nabla u_{m}(t)\right|^{2}\right)\left|\Delta u_{m}(t)\right|^{2}\right\}+2\left|a^{1 / 2} \nabla u_{m}^{\prime}(t)\right|^{2}= \\
& =-2\left(u_{m}^{\prime}(t) \nabla a, \nabla u_{m}^{\prime}(t)\right)+2\left(\nabla u_{m}^{3}(t), \nabla u_{m}^{\prime}(t)\right)+2\left(\nabla f(t), \nabla u_{m}^{\prime}(t)\right) \\
& +\left(\frac{d}{d t}\left|\nabla u_{m}(t)\right|^{2}\right)\left|\Delta u_{m}(t)\right|^{2}
\end{aligned}
$$

En adelante las letras $\alpha_{i}$ y $\beta_{j}$ representan constantes positivas.

Integrando de 0 a $t$ obtenemos:

$$
\begin{aligned}
& \left|\nabla u_{m}^{\prime}(t)\right|^{2}+\left(1+\left|\nabla u_{m}(t)\right|^{2}\right)\left|\triangle u_{m}(t)\right|^{2}+a_{0}^{1 / 2} \int_{0}^{t} \cdot\left|\nabla u_{m}^{\prime}(t)\right|^{2} d s \\
& \leq\left|\nabla u_{1}\right|^{2}+\left(1+\left|\nabla u_{0}\right|^{2}\right)\left|\triangle u_{0}\right|^{2}+\alpha_{0} \int_{0}^{t}\left|a^{1 / 2} u_{m}^{\prime}(s)\right|^{2} d s+ \\
& \alpha_{1} \int_{0}^{t}\left|\triangle u_{m}(s)\right|^{2}\left|\nabla u_{m}^{\prime}(s)\right| d s+\frac{3}{a_{0}} \int_{0}^{t}\|f(t)\|^{2} d t+\alpha_{2} \int_{0}^{t}\left|\Delta u_{m}(s)\right|^{6} d s \\
& \leq\left|\nabla u_{1}\right|^{2}+\left(1+\left|\nabla u_{0}\right|^{2}\right)\left|\triangle u_{0}\right|^{2}+\frac{3}{a_{0}} \int_{0}^{t}\|b(t)\|^{2} d t+\alpha_{0} \alpha^{2}+ \\
& \alpha_{1} \int_{0}^{t}\left|\triangle u_{m}(s)\right|^{2}\left|\nabla u_{m}^{\prime}(s)\right| d s+\alpha_{2} \int_{0}^{t}\left|\triangle u_{m}(s)\right|^{6} d s
\end{aligned}
$$

Por otro lado haciendo $w=-\triangle u_{m}(t)$ en (3.7) resulta:

$$
\begin{aligned}
& \frac{d}{d t}\left\{\left(u_{m}^{\prime}(t),-\triangle u_{m}(t)+\frac{1}{2}\left|a^{1 / 2} \nabla u_{m}(t)\right|^{2}\right\}+\left(1+\left|\nabla u_{m}(t)\right|^{2}\right)\left|\Delta u_{m}(t)\right|^{2}\right. \\
& =\left|\nabla u_{m}^{\prime}(t)\right|^{2}-\left(u_{m}^{\prime}(t) \nabla a, \nabla u_{m}(t)\right)+\left(\nabla u_{m}^{3}(t), \nabla u_{m}(t)\right)+\left(f(t),-\triangle u_{m}(t)\right)
\end{aligned}
$$

Ahora integrando de 0 o $t$ 


$$
\begin{aligned}
& \left(\nabla u_{m}^{\prime}(t), \nabla u_{m}(t)\right)+\frac{1}{2}\left|a^{1 / 2} \nabla u_{m}(t)\right|^{2}+\int_{0}^{t}\left|\Delta u_{m}(s)\right|^{2} d s \leq\left\|u_{1}\right\|\left\|u_{0}\right\|+ \\
& +\frac{1}{2}|a|_{\infty}\left\|u_{0}\right\|^{2}+\int_{0}^{t}\left|\nabla u_{m}^{\prime}(s)\right|^{2} d s+\beta_{1} \int_{0}^{t}\left|a^{1 / 2} u_{m}^{\prime}(s)\right|^{2} d s+\frac{1}{4} \int_{0}^{t}\left|\Delta u_{m}(s)\right|^{2} d s \\
& +\beta_{2} \int_{0}^{t}\left|\Delta u_{m}(s)\right|^{4} d s+\int_{0}^{t}|f(t)|^{2} d t+\frac{1}{4} \int_{0}^{t}\left|\Delta u_{m}(s)\right|^{2} d s \\
& (3.23) \quad\left(\nabla u_{m}^{\prime}(t), \nabla u_{m}(t)\right)+\frac{1}{2}\left|a^{1 / 2} \nabla u_{m}(t)\right|^{2}+\frac{1}{2} \int_{0}^{t}\left|\Delta u_{m}(s)\right|^{2} d s \\
& \leq\left\|u_{1}||\right\| u_{0}\left\|+\frac{1}{2}|a|_{\infty}\right\| u_{0} \|^{2}+\int_{0}^{t}\left|\nabla u_{m}^{\prime}(s)\right|^{2} d s+\beta_{1} \alpha^{2}+\int_{0}^{T}|f(t)|^{2} d t \\
& +\beta_{2} \int_{0}^{t}\left|\Delta u_{m}(s)\right|^{4} d s
\end{aligned}
$$

Pero notemos que para $\epsilon<\min \left\{a_{0}^{1 / 2}, a_{0}\right\}$.

$$
\begin{aligned}
& \left|\nabla u_{m}^{\prime}(t)\right|^{2}+\left|\Delta u_{m}(s)\right|+\left(a_{0}^{1 / 2}-\epsilon\right) \int_{0}^{t}\left|\nabla u_{m}^{\prime}(s)\right|^{2^{*}}+\epsilon\left(\nabla u_{m}^{\prime}(t), \nabla u_{m}(t)\right) \\
& +\frac{a_{0}}{2}\left|\nabla u_{m}\right|^{2} \geq \theta_{0}\left(\left|u_{m}^{\prime}(t)\right|+\left|\Delta u_{m}(t)\right|^{2}\right)
\end{aligned}
$$

para algún $\theta_{0}>0$.

Luego de (3.22), (3.23) y (3.24) se obtiene:

$$
\begin{aligned}
& \theta_{0}\left(\left|\nabla u_{m}^{\prime}(t)\right|^{2}+\left|\Delta u_{m}(t)\right|^{2}\right) \leq\left\|u_{1}\right\|\left\|u_{0}\right\|+\frac{1}{2}|a|_{\infty}\left\|u_{0}\right\|^{2}+\beta_{1} \alpha^{2} \\
& +\int_{0}^{T}|f(t)|^{2} d t+\left\|u_{1}\right\|^{2}+\left(1+\left\|u_{0}\right\|^{2}\right)\left|\Delta u_{0}\right|^{2}+\frac{3}{a_{0}} \int_{0}^{T}\|f(t)\| d t \\
& \int_{0}^{t}\left(\alpha_{1}\left|\nabla u_{m}^{\prime}(s)\right| d s+\alpha_{2}\left|\Delta u_{m}(s)\right|^{4}+\varepsilon \beta_{2}\left|\Delta u_{m}(s)\right|^{2}-\epsilon\right)\left|\Delta u_{m}(s)\right|^{2} d s
\end{aligned}
$$

Sea:

$F_{0}=\left\|u_{1}\right\|\left\|u_{0}\right\|+\frac{1}{2}|a|_{\infty}\left\|u_{0}\right\|^{2}+\beta_{1} \alpha^{2}+\int_{0}^{T}|f(t)|^{2} d t$

$+\frac{3}{a_{0}} \int_{0}^{T}\|f(t)\|^{2} d t+\left\|u_{1}\right\|^{2}+\left(1+\left\|u_{0}\right\|^{2}\right)\left|\Delta u_{0}\right|^{2}$

Impongamos la condición:

$$
\alpha_{1}\left|\nabla u_{1}\right|+\alpha_{2}\left|\Delta u_{0}\right|^{4}+\epsilon \beta_{2}\left|\triangle u_{0}\right|^{2} \leq \frac{\epsilon}{4}
$$




$$
\alpha_{1}\left(\frac{F_{0}}{\theta_{0}}\right)^{1 / 2}+\alpha_{2}\left(\frac{F_{0}}{\theta_{0}}\right)^{2}+\epsilon \beta_{2} \frac{F_{0}}{\theta_{0}}<\frac{\epsilon}{2}
$$

De (3.26) y (3.27), existe un $t^{*} \in\left[0, T_{m}[\right.$ tal que:

$$
\begin{aligned}
& \alpha_{1}\left|\nabla u_{1}^{\prime}\left(t^{*}\right)\right|+\alpha_{2}\left|\triangle u_{m}\left(t^{*}\right)\right|^{4}+\epsilon \beta_{2}\left|\triangle u_{m}\left(t^{*}\right)\right|^{2}<\frac{\epsilon}{2}, \forall t \in\left[0, t^{\star}[\right. \\
& \alpha_{1}\left|\nabla u_{m}^{\prime}\left(t^{\star}\right)\right|+\alpha_{2}\left|\triangle u_{m}\left(t^{\star}\right)\right|^{4}+\epsilon \beta_{2}\left|\triangle u_{m}\left(t^{\star}\right)\right|^{2}=\frac{\epsilon}{2}
\end{aligned}
$$

Seguimos de (3.25) con $t=t^{*}$ y (3.29) que:

$$
\left|\nabla u_{m}\left(t^{\star}\right)\right|^{2}+\left|\Delta u_{m}\left(t^{\star}\right)\right|^{2} \leq \frac{F_{0}}{\theta_{0}}
$$

Pero, entonces de (3.27) y (3.28) se sigue que:

$$
\begin{aligned}
& \alpha_{1}\left|\nabla u_{m}^{\prime}\left(t^{\star}\right)\right|+\alpha_{2}\left|\Delta u_{m}\left(t^{\star}\right)\right|^{4}+\epsilon \beta_{2}\left|\Delta u_{m}\left(t_{*}^{\star}\right)\right|^{2} \leq \\
& \leq \alpha_{1}\left(\frac{F_{0}}{\theta_{0}}\right)^{1 / 2}+\alpha_{2}\left(\frac{F_{0}}{\theta_{0}}\right)^{2}+\epsilon \beta_{2} \frac{F_{0}}{\theta_{0}}<\frac{\epsilon}{2} .
\end{aligned}
$$

Lo que contradice a (3.29).

La estimativa para $\left|u_{m}^{\prime \prime}(t)\right|$, se obtiene acotando primero $\left|u_{m}^{\prime \prime}(0)\right|$, derivando la ecuación aproximada (3.7), substituyendo aquí $w=2 u_{m}^{\prime \prime}(t)$, y finalmente aplicando el Lema de Gronwall.

Todas la anteriores estimativas permiten el pasaje al limite, verificando la existencia de la solución.

La verificación de las condiciones iniciales es de manera standard.

\section{BIBLIOGRAFÍA}

[1] J. R. FEITOSA, S. M. DURAES Some Remarks on the Global Existence of Solutions and Energy Decay For a Class of Hyperbolic Equations, SBA, 46 Seminario Brasileiro, 1997. p. 556 -572 .

[2] T. MASUYAMA, R. IKEHATA, Energy Decay for the Wave Equations of Kirchoff type with linear damping terms Math, Japanics, 45, 2 (1997) p.315 - 335.

[3] K. ONO, Global Existence Decay and Blowup of Solutions for Some Midly Degenerate Nonlinear Kirchoff String, J. of Diff. Eq. 137, 273 -301, (1997).

[4] L. TARTAR, Topics in non linear Analysis, Publications Mathematiques D'Orsay. 